\title{
Development Ideas of Dinosaur Resources in Animation Culture
}

\author{
Dengming $\mathrm{Gao}^{1, \mathrm{a}}$ \\ ${ }^{1}$ Zhang Daqian School of Fine Arts, Neijiang Normal University \\ ${ }^{\mathrm{a}}$ email
}

Keywords: Dinosaur, Animation culture, Development ideas

\begin{abstract}
China has rather abundant dinosaur resources and widely distributed fossil with both centralized fossil communities and fine individual species. Through scientific archaeological findings, China has the world big star dinosaurs and unique small dinosaurs. Although we have rich dinosaur resources, it seems that in the current domestic Dinosaur Museums where store the dinosaur fossils, the most displayed are simple graphics, fossils documents, and the recovered dinosaur models have very uneven qualities. In "Cultural Power, Technological Innovation" today, it is necessary to make full use of our unique fossils resources and develop science spirit, and based on the emerging animation industry, to develop the dinosaur cultural products from a unique perspective, deduce, pack, present and spread Chinese dinosaur culture in the form of animation, and display our fine cultural resources, as well as develop the cultural products with independent intellectual property rights, so as to strive to achieve the broader dinosaur cultural knowledge popularity and market achievements transformation.
\end{abstract}

\section{Importance of Dinosaur Resources Development}

Our dinosaur resources are very rich, which have been brought high attention by domestic and foreign experts. For example, Yunnan Chengjiang Fauna is the world's first specific burial fossils coenosis in Cambrian Age, and it is also one of the most important fossil treasures for the world to study the explosion evolution events in Cambrian Age; [1] The findings of fossils in Liaoning Rehe fauna has important significance to the study of the origin of birds and dinosaurs; according to our dinosaur fossil distribution, early Jurassic small and medium-sized Prosauropoda dinosaurs and middle Jurassic medium and large-sized sauropod dinosaurs are mainly founds in southwest China; at late Jurassic Age, sauropods dinosaurs reached its peak, and the giant sauropod dinosaurs were distributed in most areas of China; at early Cretaceous Epoch, Ornithopoda and Theropoda dinosaurs were mainly distributed in the central, northwest, east China; at late Cretaceous Epoch, dinosaur fauna covered the entire central and eastern regions [2]. The archaeological excavations of these dinosaur fossils communities and fossils kinds reflect the huge amount of dinosaurs and other ancient animal resources, prominent brand and recognition features, and great development and propaganda value. 


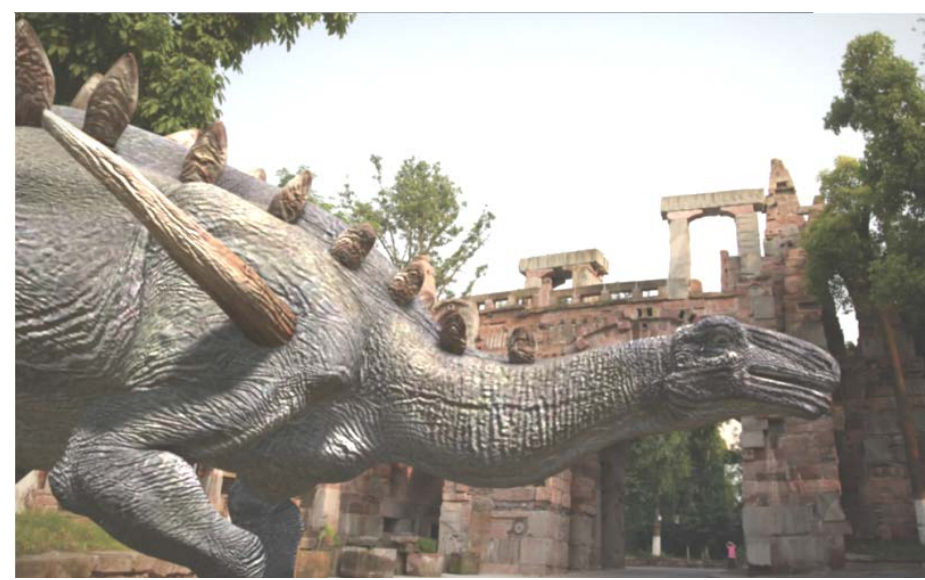

Name: ecological map of taibaii huayangosaurus Author: Dengming Gao School: Neijiang Normal University

\section{Development Ideas Analysis of Dinosaurs Recovery in Animation Culture}

Fun Animation Picture Books Design of Dinosaurs. Animation story picture book is a form of expression favored by a vast number of young people, and has a large market in the field of cultural books communication. Fun animation picture books will take dinosaurs as raw materials to carry out related story animation drawings, so as to make dinosaur picture books scientific and fun. Before the picture books are designed, the scripts shall be designed as they are the basis for a comic work and also the key element to decide its success. We can borrow the archaeological discoveries to tell the dinosaur stories; explain dinosaurs in a way of science popularization based on their characteristics, and display the dinosaurs' species and properties; besides, stories can be created based on dinosaurs' life and the relationship between dinosaurs and the same species or other species. Except the writing of the individual and the community, the major incidents in the process of biological evolution can also be used as clues to tell the public about the evolution of dinosaurs, and display the origin, development and extinction of dinosaurs on earth. In short, we can focus on the existing fossil resources for the creation, and connect various scenarios in Dinosaur Ara to write science dinosaur animation scripts.

Based on the design of a good script, fun dinosaur picture books will build related roles according to the plot. The actor shall have color relevance and graphics similarities to constitute a harmonious picture style. Based on the fixed plot and story, the designer can designs different fragments, and then form a storyboard draft with a certain visual language pattern, and finalize the script after modifications to the roles and pictures. The final draft can be in the form color and monochrome, and the visual style is also different, but the association should be paid attention to before and after the finalization. Fun dinosaur animation picture book is a re-creation to dinosaur resources and has good national cultural inheritance. At the same time, a good Chinese dinosaur animation book can lay foundation for the unique film and television and animation development, and promote industrial development in the dinosaur animation system.

Scientific Three-dimensional Dinosaur Model Design. With the development of society and the progress of science and technology, art and technology are getting more and more associated, blended and promoted by each other. The combination of art and technology enhances the innovation of national culture. In the Big Data era, art is more widely and deeply applied in the field of science, and provide conditions for analytical data and production model of dinosaurs. In recent years, thanks to the combination of high technology and art, many cultural dinosaur products are created, making the science atmosphere more active. 
Currently, 3D technology has been widely used in various industries, including the research and application of dinosaurs. The design of three-dimensional model of dinosaurs primarily base on the scientific research, and get presented in a rich art form. Three-dimensional technology enriches dinosaur images with life forms, more importantly, it makes huge dinosaur fossils assembly into a new three-dimensional database. By editing and combing, these databases can play a role in the science popularity and query of dinosaur, and make 3D technology have innovative expressions in the field of dinosaur rehabilitation.

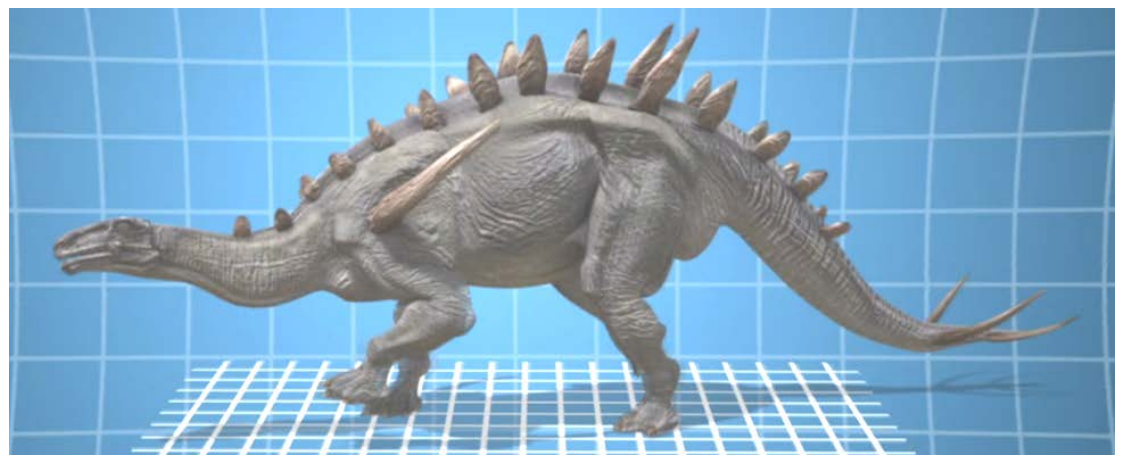

Name: design sketch of taibaii huayangosaurus Author: Dengming Gao School: Neijiang Normal University

The specific design of three-dimensional model of the dinosaur starts by making the reference pictures. By processing the fossil images and data via graphics software, making the bones, joints and other scientific data into form scale drawing, we usually create graphics from different perspectives such as front view and top view, and then apply related calculations, combination principles to correct it to obtain three-dimensional plan. The next is about the construction of the three-dimensional model. In the making process, we import data plan, adopt the polygonal modeling, subdivision modeling for basic modeling. Usually the modeling process is based on an overall start and a local shape afterwards. For the details, a high model is required, as it is the key to making a dinosaur model and also the most important part. Its main purpose is to present a more realistic reproduction of the body and objective shaping of life forms. Besides from it, the model map. Mapping is the dotting part of the entire dinosaur 3D modeling work. We usually attach a certain basis of dinosaur skin, hair, etc to the three-dimensional model based on relevant scientific information and the laws of life in order to present immersive effects. Finally, by making several three-dimensional dinosaur models, we make preparation for building a model database with a function of science popularity, such as label classification of models, texture mapping and some other sorting work.

Dinosaur Animation Development. Dinosaur animation development is a research, display way of history representation, and also a unique cultural symbol, which can provide better service for "Internet + " related dinosaur protection and promotion. The making of dinosaur animation aims to study and research the protection of dinosaur fossil resources, and apply the latest information technology into animation creation. Through digital technology, the texts, images, videos, sounds and other medias are integrated, and based on the mass media, it' ll provide dinosaur knowledge popularity services for artifacts, dinosaur museums, and other units in the field of cultural studies. Dinosaur animation making is not only able to overcome the time, space and regional restrictions of traditional display of dinosaurs, but also provide more subtle cultural guidance and knowledge services to the public.

In the Information Age, the creation and dissemination of animation has become an important way of cultural development and dissemination of information resources, and provided an important 
way for people to learn and understand dinosaur knowledge.

Dinosaur museums and animal houses, as a part of the public resources and services, have a large amount of dinosaur resources storage, which can better support the resource development. Based on the museum entities, we can take full advantage of digital technology, and through the dinosaur animation making, we fully discover the dinosaur resource information, and organically combine the creation with display, so as to play the video in public, make it as an important content and carrier, to promote public information circulation of natural resources and science popularization of dinosaur knowledge.

A small number of children dinosaur cartoons in China have been published and playing its unique features, laying foundation for the science cultural transmission. However, some insufficiencies have been found after an investigation. First, there is very few dinosaur video with research value which is telling the real story of dinosaurs, their lives and living environment. Second, scare works in line with the life characteristics of life or with certain realistic features have been created. Third, it seems difficult to find related works that are interpreting China' s dinosaur species and having international influence. Therefore, the development of independent intellectual property rights and creation of Chinese brand dinosaur animation are significantly imperative.

Peripheral Product Design of Dinosaur Animation. The peripheral product design of dinosaur animation shall firstly highlight its aesthetic and scientific features and systematically analyze roles, functions, forms, symbols and themes of dinosaur products, and secondly take cartoon derivative product development as the goal, aesthetic appreciation and science as the content-oriented organization design, to break the single-product dinosaur model making, and build systematic product design cases. The design work will be conducted according to its category after basic grasp of product design capability. Furthermore, the product design commercialization, and the market promotion shall be considered. Preferably, we do preparatory work for the design and implement the labor divisions in the dinosaur product design process. The overall production process of derivative products, the creativity, design skills and the basics design knowledge should all be paid attention to, and the multifaceted primary qualities and skills should be trained. With the principles of industry practices, we implement from the design situation, product features, product form and other aspects to improve product innovation capabilities and lay a solid foundation for animation derivative product design.

Modern animation derivative products have features of wide covering and fast propagation, but they are also an art characteristic and aesthetic symbol. When the cartoon characters has a certain popularity, the copyright owners will consider an individual or link method for further development of related industries. In the design process, we exert our imagination and creativity, master a variety of dinosaurs product picture and packaging design, to create a new unique dinosaur animation peripheral product. In the meantime, we could attract the public participation in a certain way, improve their operational and participation capacity, and promote the development of the dinosaurs in the animation industry.

\section{Significance}

Dinosaur development is an important part of scientific research and culture dissemination, and it is closely related to the development of science animation. China has put forward "Culture Innovation", "Scientific and Technological Innovation" concepts to provide a good environment for the development of cultural industries. The social development objectively urges the development of science and culture and the animation market, also contributes to the molding of dinosaur culture in the animation industry. New opportunities and challenges provide more chances and platforms 
for the public to take dinosaur fossils as blueprint, science and scientific research as the basis, to carry out the script design, 3D model design, animation creation and animation peripheral products design, and construct related materials for virtual digital museum, do theoretical groundwork for creating animation interactive industry, "Internet +" industry, and big data industry.

In addition, the development of the dinosaur animation mobilizes scientists, cartoonists, animators and other research personnel and scientific culture lovers to stand on the stage of society to launch a sports science sport with the background of animation and the element of dinosaur, so that science and art can be more integrated, cultural dinosaur deeply rooted, and China' s cultural soft power greatly increase.

\section{Acknowledgements}

Fund Project: the National Social Science Fund Project (13xk8008);

Ministry of Education, "New Century Talents Support Program" (NCET-13-0925);

This paper is funded by the below projects: Chongqing College Outstanding Achievement Transformation Project, Project Number: KJZH14106;

Sichuan Department of Education Scientific Research Project, Project Number: 16SB0164;

Sichuan Youth Ideological and Moral Construction Research Center Project, Project Number: XKQSN2015ZD04;

Neijiang Normal University Scientific Research Project, Project Number: 15JC02;

Neijiang Science Planning Project, Finance Number: 2015069.

\section{References}

[1] F. C. Zhao, M. Y. Zhu, and S. X. Hu. Yunnan Early Chengjiang Fauna Palaeocommunities at Cambrian Age [J]. Chinese Science: Earth Sciences, 2010,09: 1135-1153 + 1301-1306.(In Chinese) [2] J. R. Xu, K. Li, and C. Y. Yang. Chinese Dinosaur Fossil Resources and Evaluation [J]. Technological Management of Land Resources, 2014,02: 8-16.(In Chinese) 